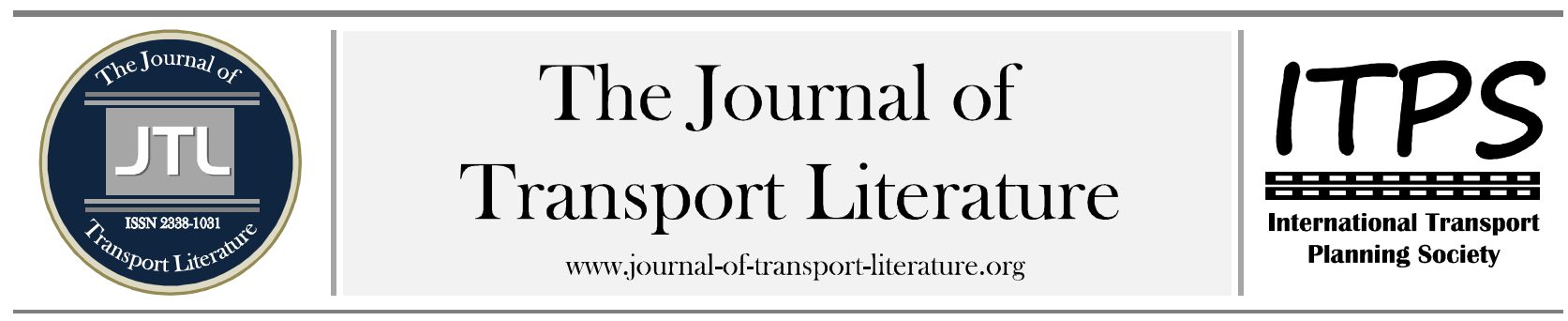

\title{
Modelo de previsão do índice de condição dos pavimentos flexíveis
}

Sérgio Pacífico Soncim ${ }^{1,+} ;$ José Leomar Fernandes Júnior ${ }^{2}$

${ }^{1}$ Universidade Federal de Itajubá, Itabira, Brasil

2 Universidade de São Paulo, São Carlos, Brasil

\section{Article Info}

Palavras-chave:

Sistemas de gerência de pavimentos

Modelos de desempenho Defeitos

Submitted 29 Oct 2014; received in revised form 15 Jan 2015 . accepted 20 Apr 2015

Licensed under

Creative Common

CC-BY $3.0 \mathrm{BR}$.

\section{Resumo}

Este trabalho apresenta o desenvolvimento de um modelo de previsão do índice de condição dos pavimentos flexíveis para a malha rodoviária, em concreto asfáltico usinado a quente, do Estado da Bahia. Teve como ponto de partida um planejamento fatorial, elaborado com base em informações de um banco de dados de rodovias, fornecido pelo Departamento de Infraestrutura de Transportes da Bahia. Para a verificação da adequação do modelo utilizou-se a reta de ajuste dos dados, o coeficiente de correlação (r), a análise de resíduos e o gráfico de probabilidade normal dos resíduos. 0 modelo correlaciona 0 desempenho do índice de condição dos pavimentos flexíveis às variáveis idade, tráfego e pluviometria, fatores que tem influência no processo de deterioração dos pavimentos. 0 modelo desenvolvido apresentou ajuste aos dados observados e $r=0,64$, mostrando indícios da validade da sua aplicação, para as características da malha rodoviária do Estado da Bahia. Não existem modelos de desempenho universais e modelos que foram desenvolvidos a partir de dados observados em estudos realizados em locais específicos não são necessariamente transferíveis, e possuem algumas limitações inerentes.

+ Corresponding author. Universidade Federal de Itajubá, Campus Itabira. Rua Irmã Ivone Drumond 200. CEP 35903087 - Itabira, MG - Brasil.

E-mail address: sergiops@unifei.edu.br.

\section{Introdução}

O objetivo deste trabalho foi desenvolver um modelo para a previsão de desempenho do índice de condição dos pavimentos flexíveis (ICPF), para a rede rodoviária em concreto asfáltico usinado a quente do Estado da Bahia. 0 trabalho teve como ponto de partida um planejamento fatorial, elaborado com base em informações de um banco de dados de rodovias, fornecido pelo Departamento de Infraestrutura de Transportes da Bahia. 0 índice de condição dos pavimentos flexíveis é um valor numérico que expressa o atual estado de condição de um pavimento, baseado no levantamento visual de defeitos.

Modelos para a previsão da condição do pavimento fazem parte de um Sistema de Gerência de Pavimentos (SGP) e são utilizados tanto em nível de rede, para planejamento, estimativa das necessidades totais de manutenção e reabilitação, priorização de projetos e programação de investimentos, como em nível de projeto, para a definição de atividades de manutenção e de reabilitação. Os modelos de previsão de desempenho devem refletir as condições às quais eles são aplicados e devem ser desenvolvidos a partir de dados locais. O Brasil representa um exemplo excelente dessa necessidade, pois possui condições climáticas diversificadas e grande variedade de solos (Queiroz, 1981). 0 planejamento das ações de manutenção depende, acima de tudo, da capacidade das agências rodoviárias de prever a condição futura do pavimento. Falhas nesse processo podem resultar em escolhas erradas de estratégias e, consequentemente, em utilização ineficiente dos recursos.

Desde a década de 70 estudos têm sido realizados no Brasil com o objetivo de desenvolvimento de modelos de desempenho para utilização em gerência de pavimentos. Merecem destaque os estudos iniciados em 1975 pela Empresa Brasileira de Planejamento de Transportes (GEIPOT, 1981) que resultaram na Pesquisa de Inter-Relacionamento de Custos de Construção, Conservação e Utilização de Rodovias (PICR).

Paterson (1987) desenvolveu as equações de desempenho de pavimentos utilizadas no The Highway Design and Maintenance Standards Model (Watanada et al., 1987). A base empírica para o desenvolvimento dos modelos de regressão foi a mesma utilizada por Queiroz (1981) durante a PICR. 0 motivo da escolha foi a grande quantidade de dados observados ao longo do tempo que incluem irregularidades, trincamento, desgaste, deformações permanentes, atividades de manutenção, tráfego e pluviometria para uma programação fatorial de trechos de pavimentos em serviço da malha rodoviária compreendida no quadrilátero Brasília, Goiânia, Belo Horizonte e São Paulo (Paterson, 1989). Marcon (1996) desenvolveu modelos de desempenho com base em dados da rede rodoviária do Estado de Santa Catarina. 0 desempenho dos pavimentos foi representado pelas variáveis dependentes quociente de irregularidade, deflexões, índice de gravidade global, trincamento total e afundamento nas trilhas de roda, tendo como variável independente o intervalo de tempo, em anos, ou o número de operações do eixo padrão de $80 \mathrm{kN}$. Foram testados cinco tipos de equações por meio de regressão. Para cada par de variáveis foi indicada a equação que apresentava o melhor coeficiente de determinação. 
Yshiba (2003) desenvolveu modelos estatísticos que representam os efeitos dos fatores idade, tráfego e número estrutural corrigido sobre o desempenho de pavimentos, quantificado em termos de irregularidade longitudinal e deflexões. A técnica usada foi a análise da variância aplicada aos dados de levantamentos realizados em 1995 e 1998 na malha rodoviária do Estado do Paraná. Benevides (2006) desenvolveu modelos estatísticos baseado em levantamentos realizados em 72 trechos da malha rodoviária pavimentada da região metropolitana de Fortaleza, em 2006. Nos modelos estatísticos foram estabelecidas equações de regressão, utilizando a análise de variância. Foram determinadas equações de evolução para os defeitos para dados coletados em 2004 e 2005, assim como equações de regressão que relacionam os valores de irregularidade longitudinal obtido com os perfilômetros inercial e a laser.

Albuquerque (2007) desenvolveu modelos de previsão de desempenho a partir de dados de levantamentos de deflexão com FWD, de deflexão com Viga Benkelman e de irregularidade longitudinal para pavimentos em tratamento superficial dos Estados do Ceará e Paraíba. 0 clima foi considerado pelo Índice de Aridez desenvolvido pela UNESCO e a capacidade estrutural do pavimento foi expressa pelo número estrutural corrigido (SNC). Já a idade do pavimento e as cargas de tráfego foram expressas em termos de número acumulado de repetições do eixo padrão. Todos os modelos foram construídos através de regressão por crescimento exponencial. Soncim (2011) e Soncim e Fernandes Jr. (2014) desenvolveram modelos de desempenho da irregularidade longitudinal e da progressão da área de trincas para dados de trechos construídos em tratamento superficial e em concreto asfáltico usinado a quente (CAUQ), da malha rodoviária do Estado da Bahia.

Os fatores considerados para o desenvolvimento do modelo foram a idade, o tráfego e a pluviometria, e a análise da variância foi a técnica utilizada para avaliar a significância dos fatores e definir os parâmetros do modelo de desempenho. Para a verificação da adequação do modelo estatístico utilizou-se a reta de ajuste dos dados previstos aos observados, com determinação do coeficiente de correlação (r), a análise de resíduos, e o gráfico de probabilidade normal dos resíduos. 0 modelo desenvolvido neste trabalho apresentou ajuste aos dados observados e coeficiente de correlação $r=0,64$, mostrando indícios da validade da sua aplicação, para as características da malha rodoviária em concreto betuminoso usinado a quente da malha rodoviária do Estado da Bahia.

A seção 1 descreve o que representa um índice combinado de defeitos e apresenta os principais índices de condição de pavimento utilizados no Brasil. A seção 2 apresenta o planejamento fatorial utilizado no desenvolvimento do modelo, estatísticas dos trechos estudados, os fatores considerados e seus níveis. A seção 3 mostra o desenvolvimento do modelo e o método utilizado para a sua validação e, por último, a seção 4 apresenta as conclusões e contribuições do trabalho, bem como sugestões de futuras pesquisas.

\section{1. Índices combinados de defeitos}

Durante a avaliação da superfície de trechos de pavimentos rodoviários é natural que sejam levantados diversos tipos de defeitos, com diferentes níveis de severidade e densidade. Isto pode gerar um número extenso de combinações que pode conduzir a determinação equivocada do atual estado de condição do pavimento avaliado. Para resolver esse problema é necessário converter os resultados dessas avaliações em um valor, ou um índice combinado de defeitos, que possa ser utilizado como critério na tomada de decisão sobre priorização de atividades de manutenção e reabilitação.

Os índices combinados de defeitos podem ser determinados através de avaliações subjetivas (feitas por painéis de avaliadores) ou calculados a partir de informações detalhadas sobre a extensão e o nível de severidade de diferentes formas de deterioração dos pavimentos. A condição do pavimento pode ser quantificada, por exemplo, pelo índice de Condição do Pavimento (ICP) (Fernandes Jr.; Oda; Zerbini, 2006). O ICP é um valor numérico que varia de 0 (para uma péssima condição) a 100 (para uma excelente condição). Sua determinação é baseada no levantamento visual de defeitos no qual os tipos de defeitos, o nível de severidade e sua densidade são anotados e medidos para uma determinada seção de pavimento, de acordo com o PCI Distress Identification Manual. 0 método é baseado na dedução de valores ponderados que variam de 0 a 100 (o pavimento já sai com 100 pontos) que irá indicar o impacto que cada defeito tem na condição do pavimento em termos de severidade e densidade. Se o valor deduzido é zero significa que o nível de manifestação corrente de um determinado defeito não apresenta impacto no atual desempenho do pavimento, caso contrário, indica grande impacto.

No Brasil, é muito utilizado o Índice de Gravidade Global (IGG), que é um índice de defeitos combinado, definido por um número que permite a avaliação das condições de deterioração apenas de uma seção ou através de comparação relativa entre várias seções de uma malha de pavimentos (Yshiba, 2003). Apesar de o IGG ser utilizado para classificar o estado geral de um determinado trecho homogêneo de pavimento, em função da incidência de defeitos de superfície, autores como (Yshiba, 2003), (Benevides, 2006) e (Fernandes Jr.; Oda; Zerbini, 2006) citam que as principais limitações do método do IGG são o fato de não levar em conta o nível de severidade, apenas o tipo de defeito (exceto para as trincas), e considerar apenas o número de ocorrências e não a extensão.

As normas DNIT 005/2003, DNIT 006/2003, DNIT 007/2003 e DNIT 008/2003 estabelecem as definições, os critérios e os equipamentos utilizados para a realização dos levantamentos de defeitos de pavimentos. Os levantamentos podem ser realizados tanto por meio de caminhamento (ou a pé) quanto por meio de método que se utiliza de avaliadores dentro de um veículo em movimento. Neste último caso chamado de levantamento visual contínuo (LVC), determinam-se o índice de condição de pavimentos flexíveis (ICPF), o Índice de Gravidade Global Expedito (IGGE) e o Índice de Estado de Superfície (IES). O ICPF é um índice obtido a partir do levantamento de defeitos da superfície dos pavimentos, como panelas, trincas etc. e seu valor numérico varia de 0 (para uma péssima condição) a 5 (para uma excelente condição).

\section{Planejamento fatorial}

O planejamento fatorial do experimento considerou os fatores idade do pavimento (ID), tráfego, representado pelo número de aplicações equivalentes do eixo-padrão desde a sua abertura até o ano de 2004 (TA), e pluviometria (PL). Foram adotados dois níveis para cada fator, obtendo-se um experimento fatorial tipo $2^{3}=8$. Houve réplicas, ou seja, repetições de avaliações em seções de pavimento pertencentes à uma mesma célula de uma matriz fatorial, cujos resultados foram obtidos com os mesmos equipamentos, equipe de campo, procedimentos e espaço de inferência. Os níveis foram escolhidos de acordo com a distribuição dos fatores idade do pavimento (ID), tráfego desde a abertura do pavimento até o ano de 2004 (TA) e pluviometria (PL), para os trechos em CAUQ que não haviam sofrido atividades de manutenção e reabilitação até o 
ano de 2004, de acordo com o banco de dados do DERBA, resultando num total de 78 trechos. A Tabela 1 apresenta, para os 78 trechos, os valores médios, mínimos e máximos para os fatores ID, TA e PL.

Tabela 1 - Valores médios, mínimos e máximos dos fatores ID, TA e PL nos 78 trechos em CAUQ

\begin{tabular}{llll}
\multicolumn{3}{c}{ Fonte: Autores } \\
\hline Fator & Média & Mínimo & Máximo \\
ID & 24 & 6 & 40 \\
TA & 2.013 .039 & 35.002 & 4.705 .610 \\
PL & 888 & 383 & 1934 \\
\hline
\end{tabular}

Entretanto, não foram utilizados todos os 78 trechos em CAUQ sem atividades de manutenção e reabilitação até 2004, pois alguns dos trechos não tinham, simultaneamente, informações da variável dependente ICPF. Os valores das réplicas utilizados para o desenvolvimento dos modelos foram escolhidos de forma aleatória, para células que apresentaram mais de dois valores. Os demais valores foram utilizados na fase da validação dos modelos. Os valores limites dos níveis dos fatores foram escolhidos de maneira que as células do arranjo do experimento fatorial fossem preenchidas com pelo menos duas possibilidades de trechos (ao menos uma réplica).

0 planejamento fatorial para os trechos em CAUQ sem atividades de manutenção e reabilitação até 2004 teve o fator

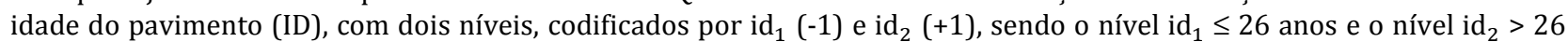
anos. 0 fator tráfego desde a sua abertura até o ano de 2004 (TA) foi codificado com dois níveis, $\operatorname{ta}_{1}(-1)$ e ta $2(+1)$, sendo o nível $\mathrm{ta}_{1} \leq 2 \times 10^{6}$ operações do eixo padrão de $80 \mathrm{kN}$ e o nível ta $\mathrm{ta}_{2}>2 \times 10^{6}$ operações do eixo padrão de $80 \mathrm{kN}$. 0 fator pluviometria (PL) foi codificado com dois níveis, $\mathrm{pl}_{1}(-1)$ e $\mathrm{pl}_{2}(+1)$, sendo o nível $\mathrm{pl}_{1} \leq 720 \mathrm{~mm} /$ ano e o nível $\mathrm{pl}_{2}>720$ $\mathrm{mm} / \mathrm{ano}$.

\section{Desenvolvimento do modelo}

A Análise de variância é um teste estatístico que visa verificar se existe uma diferença significativa entre as médias provenientes de amostras distintas e se os fatores exercem influência no modelo. A distribuição $\mathrm{F}$ é utilizada pela Análise da Variância na decisão de aceitar ou rejeitar a hipótese de igualdade entre grupos de dados (hipótese nula). 0 objetivo do

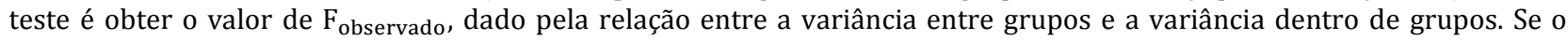

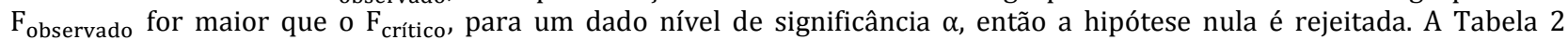
apresenta o resumo do cálculo da Análise de Variância dos fatores e suas interações, considerando-se a variável dependente irregularidade longitudinal, para um nível de significância de $5 \%\left(\alpha=0,05 ; \mathrm{F}_{\text {crítico }}=5,32\right)$.

Tabela 2: Resumo da ANOVA para o planejamento fatorial do ICPF Fonte: Autores

\begin{tabular}{|c|c|c|c|c|c|c|c|c|}
\hline Fator & $\begin{array}{l}\text { Soma } \\
\text { Quadrática }\end{array}$ & $\begin{array}{l}\text { Graus de } \\
\text { Liberdade }\end{array}$ & $\begin{array}{l}\text { Média } \\
\text { Quadrática }\end{array}$ & Efeito & Coeficiente & Fo & Significativo & $\begin{array}{l}\text { Valor } \\
\mathbf{P}\end{array}$ \\
\hline$I D$ & 2,352 & 1 & 2,352 & $-0,767$ & $-0,383$ & 12,911 & SIM & 0,007 \\
\hline$T A$ & 5,176 & 1 & 5,176 & $-1,138$ & $-0,569$ & 28,415 & SIM & 0,001 \\
\hline$P L$ & 1,210 & 1 & 1,210 & $-0,550$ & $-0,275$ & 6,643 & $\operatorname{SIM}$ & 0,033 \\
\hline$I D^{*} T A$ & 0,276 & 1 & 0,276 & 0,263 & 0,131 & 1,513 & $N \tilde{O} O$ & 0,254 \\
\hline$I D^{*} P L$ & 0,123 & 1 & 0,123 & 0,175 & 0,088 & 0,673 & $N \tilde{A} O$ & 0,436 \\
\hline$T A^{*} P L$ & 0,002 & 1 & 0,002 & 0,021 & 0,010 & 0,009 & $N \tilde{A} O$ & 0,925 \\
\hline$I D^{*} T A^{*} P L$ & 0,350 & 1 & 0,350 & $-0,296$ & $-0,148$ & 1,921 & $N \tilde{O} O$ & 0,203 \\
\hline Erro & 1,457 & 8 & 0,182 & & & & & \\
\hline Total & 10,944 & 15 & & & 2,396 & & & \\
\hline
\end{tabular}

A análise de regressão foi usada para a determinação dos parâmetros do modelo estatístico para previsão do ICPF, conforme apresentado na Equação $1\left(\mathrm{R}^{2}=0,80\right)$. As equações polinomiais correspondentes aos fatores idade do pavimento, tráfego e pluviometria são apresentadas nas Equações 2, 3 e 4.

$$
\begin{gathered}
\text { ICPF }=2,39-0,39 P(I D)-0,57 P(T A)-0,28 P(P L) \\
P(I D)=\frac{I D-23}{15} \\
P(T A)=\frac{T A-2 \times 10^{6}}{3,5 \times 10^{6}} \\
P(P L)=\frac{P L-948}{3840}
\end{gathered}
$$

onde:

$\mathrm{P}(\mathrm{ID})$ é a equação polinomial para o fator idade desde a construção;

$\mathrm{P}(\mathrm{TA})$ é a equação polinomial para o fator tráfego;

$\mathrm{P}(\mathrm{TA})$ é a equação polinomial para o fator pluviometria;

ID é o valor quantitativo da variável Idade do pavimento, em anos;

TA é o valor quantitativo da variável tráfego, em número de aplicações equivalentes do eixo-padrão;

PL é o valor quantitativo da variável pluviometria, em mm anuais. 
Para a verificação da adequação do modelo estatístico utilizou-se a reta de ajuste dos dados previstos aos observados, com determinação do coeficiente de correlação (r) correspondente (Figura 1), a análise de resíduos (Figura 2), e o gráfico de probabilidade normal dos resíduos (Figura 3). Deve-se destacar que o conjunto de dados observados, utilizados para validação do modelo, também foram coletados na etapa de levantamento de campo, em seções de pavimentos diferentes das que foram consideradas para o desenvolvimento do modelo de previsão de desempenho. As Figuras 1, 2 e 3 apresentam, respectivamente, a equação de correlação linear com o coeficiente de correlação, o gráfico de resíduos e o gráfico de distribuição de probabilidade normal dos resíduos. Pela análise da Figura 1 pode-se perceber que a reta de regressão linear do ICPF apresentou bom ajuste ao conjunto de pares de pontos observados e previstos, com $r=0,64$. Entretanto, a observação da reta de regressão com o intercepto igual a 0 indica que ocorre a tendência de superestimação para menores valores e subestimação para maiores valores observados do ICPF. A Figura 2 mostra que existe uma distribuição aleatória em torno de zero, o que indica a igualdade entre as variâncias, enquanto que a Figura 3 indica a normalidade dos dados, pois os resíduos estão distribuídos aproximadamente ao longo de uma linha reta.

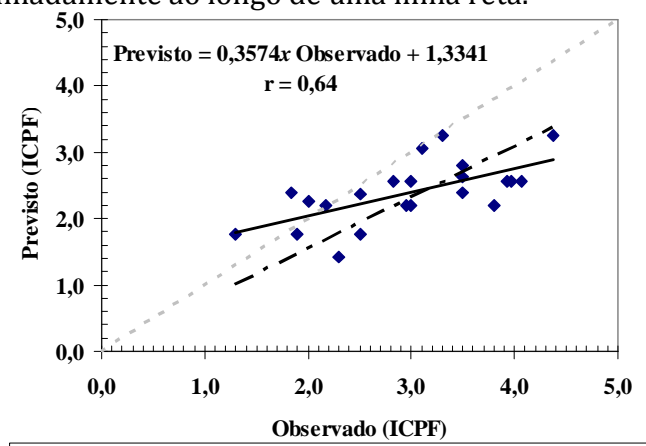

- ICPF L Linear ICPF - - Linear ICPF intercepto = 0

Figura 1 - Correlação dos valores observados do ICPF com os valores previstos Fonte: Autores

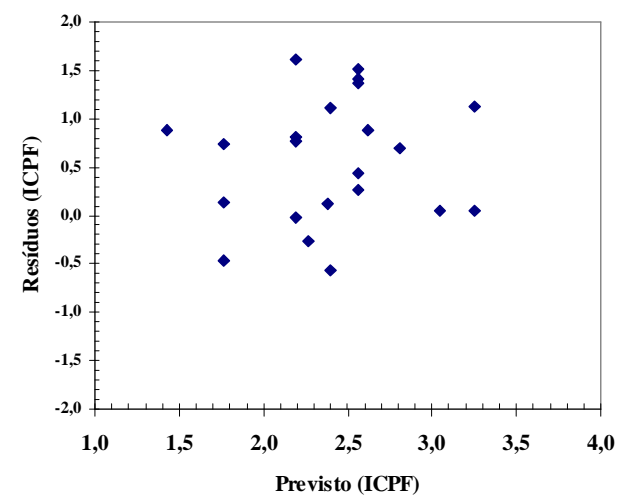

Figura 2 - Gráfico de resíduos do modelo de desempenho do ICPF obtido

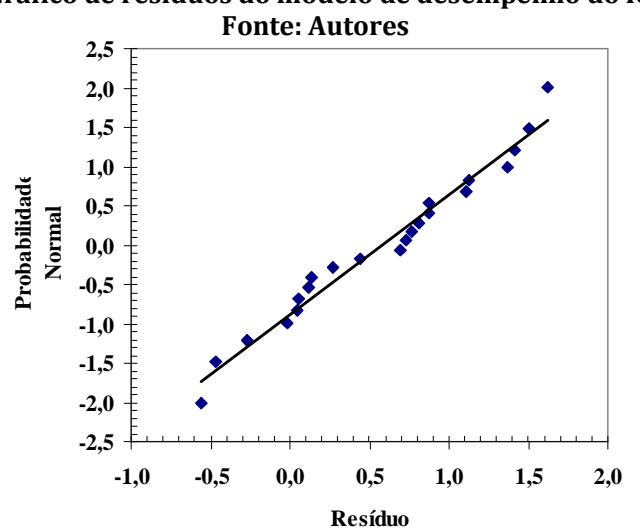

Figura 3 - Gráfico de distribuição de probabilidade dos resíduos do ICPF Fonte: Autores

\section{Conclusão}

Foi desenvolvido um modelo de desempenho para previsão do índice de condição dos pavimentos flexíveis, considerando dados da malha rodoviária pavimentada em concreto asfáltico usinado a quente do Estado da Bahia. 0 modelo desenvolvido neste trabalho apresentou ajuste aos dados observados e coeficiente de correlação $r=0,64$. Não foi possível a comparação com outros modelos de desempenho do índice de condição dos pavimentos flexíveis de pavimentos asfálticos desenvolvidos para outras redes viárias brasileiras constituídas por revestimentos em concreto asfáltico usinado a quente, ou outro tipo de revestimento, pois não foram encontrados quando da revisão bibliográfica. 0 modelo desenvolvido correlaciona o desempenho do índice de condição dos pavimentos flexíveis, utilizado para gerência de pavimentos em 
organismos rodoviários, às variáveis idade do pavimento, tráfego e pluviometria, consideradas fatores que tem influência no processo de deterioração dos pavimentos, em gerência em nível de rede.

0 modelo foi desenvolvido para os dados e características da malha rodoviária do Estado da Bahia, portanto, sua aplicabilidade limita-se às condições as quais o mesmo foi desenvolvido. 0 modelo poderá ser aplicado a outras malhas rodoviárias, condicionado a sua calibração para as condições de tipo de estrutura, tráfego, clima e idade dos pavimentos de outras regiões do Brasil. Para que se obtenham melhores resultados para a previsão do desempenho dos pavimentos da rede de rodovias pavimentadas do Estado da Bahia é necessário que sejam realizados novos levantamentos para alimentação do banco de dados, de forma que se possa melhorar a precisão e acurácia do modelo desenvolvido.

Há a necessidade de estudos complementares, que incluam fatores que não puderam ser considerados neste trabalho, como a capacidade estrutural do pavimento e informações relacionadas à drenagem, por exemplo. Para tanto, o método utilizado, baseado no planejamento fatorial e na Análise de Variância, poderá ser repetido, pois se mostrou capaz de definir as quantidades dos dados para a realização do estudo, sem comprometer a precisão e acurácia do modelo de desempenho obtido.

Sugere-se a comparação do modelo de desempenho desenvolvido neste trabalho com os modelos de deterioração do HDM-4, por meio da aplicação do banco de dados do DERBA. É necessário, também, que sejam desenvolvidos fatores de calibração a serem incluídos nos modelos de deterioração do HDM-4, considerando as características da malha rodoviária pavimentada do Estado da Bahia e de forma que possam utilizar os resultados obtidos com o modelo de previsão de desempenho desenvolvido nesta pesquisa.

\title{
Referências
}

Albuquerque, F. S. (2007) Sistema de gerência de pavimentos para departamentos de estradas do nordeste brasileiro. (Tese de Doutorado). Universidade Federal do Rio Grande do Sul, Porto Alegre, Brasil.

Benevides, S. A. S. (2006) Modelos de desempenho de pavimentos asfálticos para um sistema de gestão de rodovias estaduais do Ceará. (Tese de Doutorado). COPPE, Universidade Federal do Rio de Janeiro, Rio de Janeiro, Brasil.

Departamento Nacional de Infraestrutura de Transportes (2003). Defeitos nos pavimentos flexíveis e semi-rígidos (DNIT - 005/2003 TER).

Departamento Nacional de Infraestrutura de Transportes (2003). Avaliação objetiva da superfície de pavimentos flexíveis e semi-rígidos (DNIT - 006/2003 - PRO).

Departamento Nacional de Infraestrutura de Transportes (2003). Levantamento para a avaliação da condição de superfície de subtrecho homogêneo de rodovias de pavimentos flexíveis e semi-rígidos para a gerência de pavimentos e estudos e projetos (DNIT 007/2003 - PR0).

Departamento Nacional de Infraestrutura de Transportes (2003). Levantamento visual contínuo para a avaliação da superfície de pavimentos flexíveis e semi-rígidos (DNIT - 008/2003 - PRO).

Fernandes Jr., J. L.; \& Oda, S.; \& Zerbini, L. F. (2006). Defeitos e atividades de manutenção e reabilitação em pavimentos asfálticos. $101 \mathrm{f}$. Gráfica da Escola de Engenharia de São Carlos, Universidade de São Paulo, São Paulo.

GEIPOT (1981). Pesquisa Sobre o Inter-relacionamento dos Custos de Construção, Conservação e Utilização de Rodovias. Empresa Brasileira de Planejamento de Transportes, Ministério dos Transportes, Brasília, DF.

Marcon, F. M. (1996). Contribuição ao desenvolvimento de um sistema de gerência de pavimentos para a malha rodoviária estadual de Santa Catarina. (Tese de Doutorado). Centro Técnico aeroespacial, Instituto Tecnológico de Aeronáutica, São Paulo, Brasil.

Paterson, W. D. O. (1987). Road Deterioration and Maintenance Effects: Models for Planning and Management. Published for the Word Bank, Washington, D.C.

Paterson, W. D. O. (1989). A transferable causal model for predicting roughness progression in flexible pavements. Transportation Research Record, Washington, 1215, 70-84.

Queiroz, C. A. V (1981). Performance prediction models for pavement management in Brazil. (Doctoral dissertation). The University of Texas at Austin, Texas, US.

Soncim, P. S. (2011). Desenvolvimento de modelos de previsão de desempenho de pavimentos asfálticos com base em dados da rede de rodovias do Estado da Bahia. (Tese de Doutorado). Escola de Engenharia de São Carlos, Universidade de São Paulo, São Paulo, Brasil.

Soncim, P. S.; \& Fernandes Jr., J. L. (2014). Modelo de previsión de la progression del área de fisuras em pavimentos asflaticos. Journal of Transport Literature, 8 (2), 221-243.

Yshiba, J. K. (2003). Modelos de previsão de desempenho: estudos de rodovias do Estado do Paraná. (Tese de Doutorado). Escola de Engenharia de São Carlos, Universidade de São Paulo, São Paulo, Brasil.

Watanada, T.; \& Paterson, W.; \& Bhandi, A.; \& Harral, C.; \& Dhareshwar, A. M.; \& Tsunokawa, K. (1987). The Highway Design and Maintenance Standards Model. v.1.

\begin{abstract}
This paper presents the development of a predicted performance model for a flexible pavement condition index for a network in hot asphalt concrete mix in the state of Bahia. Its starting point was a factorial design based on information from a database of highways, provided by the Department of Transport Infrastructure of Bahia. To verify the adequacy of the statistical model was used the straight fit to the observed data set, with determination of the correlation coefficient ( $\mathrm{r}$, residue analysis, and normal probability plot of the residuals. The model correlates the performance of a pavement condition index, used for pavement management, to pavement age, traffic and rainfall, factors considered having influence on the pavement deterioration process. The model developed has fit the observed data and showed $\mathrm{r}=$ 0.64 , so that, there is evidence of the validity of its application for the road network of Bahia. There are no universal performance models and models that have been developed from data observed in studies in specific locations are not necessarily transferable, and have some inherent limitations.
\end{abstract}

Key words: pavements management systems, performance models, distresses. 